\title{
Direct detection of concerted proton and electron transfer in human manganese superoxide dismutase
}

\author{
Gloria BorgstahI ${ }^{1}$, Jahaun Azadmanesh ${ }^{1}$, Will Lutz ${ }^{1}, K_{\text {Kevin Weiss }}{ }^{2}$, Leighton Coates ${ }^{2}$ \\ ${ }^{1}$ University of Nebraska Medical Center, Omaha, Nebraska, United States of America; \\ ${ }^{2}$ Oak Ridge National Laboratory, Tennessee, United States of America \\ gborgstahl@unmc.edu
}

Superoxide dismutases (SODs) are the major regulators of oxidative stress and therefore the first line of defense to protect organisms against metabolic- and environmentally-induced reactive oxygen species (ROS). Human mitochondrial manganese SOD (MnSOD) expression is modulated to prevent ROS-based damage, promote redox homeostasis and maintain proper cell signaling. Our research goal is to understand the molecular basis of how MnSOD uses coupled proton-electron transfers to dismute superoxide. For this, the 3D arrangement of all atoms is needed, most importantly the position of protons. Our recent technical advancements with neutron crystallography at Oak Ridge National Laboratory have overcome the limitations of X-ray crystallography - revealing proton positions with high detail while also allowing control of the metal electronic state. In this research project, MnSOD neutron maps reveal the proton relays to the active site metal and the protonation states of metal-bound ligands. Our results demonstrate the transfer of protons to the bound active site solvent that is triggered by the reduction of the active site manganese. This proton transfer involves unusual active site amino acid pKas, at least five low barrier hydrogen bonds, glutamine tautomerization and a water bridge in the active site channel.

Keywords: superoxide dismutase, metalloenzyme, oxidoreductase, oxidation/reduction, proton transfer 